\title{
Intervention and Mediation Effects of Target Processes in a Randomized Controlled Trial of Acceptance and Commitment Therapy for Anxious Cancer Survivors in Community Oncology Clinics
}

\author{
Joel N. Fishbein ${ }^{\mathrm{a}^{*}}$ \\ Charles M. Judd ${ }^{\mathrm{a}}$ \\ Sarah Genung ${ }^{\mathrm{a}}$ \\ Annette L. Stanton ${ }^{\mathrm{b}, \mathrm{c}, \mathrm{d}, \mathrm{e}}$ \\ Joanna J. Arch a,f
}

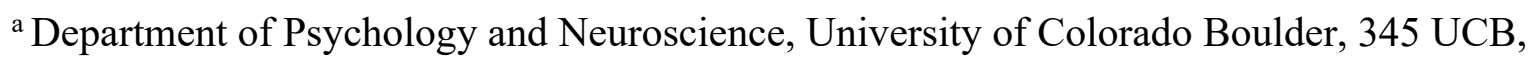

Boulder, CO, 80309, USA

${ }^{\mathrm{b}}$ Department of Psychology, University of California, Los Angeles, 502 Portola Plaza, Los Angeles, CA, 90095, USA

${ }^{\mathrm{c}}$ Jonsson Comprehensive Cancer Center, University of California, Los Angeles, 10833 Le Conte Ave, Los Angeles, CA 90024, USA

${ }^{\mathrm{d}}$ Cousins Center for Psychoneuroimmunology, University of California, Los Angeles, 300

Medical Plaza Driveway, Los Angeles, CA 90095, USA

${ }^{\mathrm{e}}$ Department of Psychiatry and Biobehavioral Sciences, University of California, Los Angeles, 757 Westwood Plaza \#4, Los Angeles, CA 90095, USA

${ }^{\mathrm{f}}$ Division of Cancer Prevention and Control, University of Colorado Cancer Center, 1665 Aurora Court, Aurora, CO 80045, USA

*Corresponding author: joel.fishbein@,colorado.edu 


\section{Author Note}

Funding: The Valued Living study, which provided the dataset analyzed in this manuscript, was supported by an American Cancer Society Research Scholar Grant (PI: Arch, RSG-15-020-01-CPPB). Dr. Arch also receives unrelated research funding from AstraZeneca. 


\begin{abstract}
Objective: Acceptance and Commitment Therapy (ACT) is a promising psycho-oncological intervention, but its mechanisms in real-world settings are not fully understood. This study examined core theorized ACT and broader ACT-consistent target processes as mediators of ACT versus minimally-enhanced usual care within a randomized trial for anxious cancer survivors in a community oncology setting.
\end{abstract}

Method: Two core theorized ACT target processes (experiential avoidance and values-aligned behavior, each measured with two instruments) and two broader ACT-consistent target processes (emotional approach coping and self-compassion) were analyzed at pre- and post-intervention as mediators of general anxiety symptoms, cancer-related trauma symptoms, and fear of cancer recurrence $(N=134)$.

Results: ACT led to greater increases on emotional approach coping $(p s \leq .001)$ and one measure of values-aligned behavior ( $p s \leq .031)$, and marginal or greater improvement on selfcompassion $(p \mathrm{~s} \leq .055)$, but not other core ACT target processes. Self-compassion and emotional approach coping mediated ACT's effects on cancer-related trauma symptoms ( $p \mathrm{~s} \leq .037)$. Additionally, self-compassion, emotional approach coping, and values-aligned behavior marginally mediated fear of recurrence and general anxiety symptoms improvement $(p s \leq .088)$. Conclusion: ACT reduced cancer survivors' anxiety-related symptoms, and especially cancerrelated trauma symptoms, most consistently by promoting self-compassion and emotional approach coping.

Keywords: Acceptance and Commitment Therapy; Anxiety; Neoplasms; Experiential Avoidance; Self-compassion; Coping Style 


\section{Intervention and Mediation Effects of Target Processes in a Randomized Controlled Trial of Acceptance and Commitment Therapy for Anxious Cancer Survivors in Community Oncology Clinics}

Acceptance and Commitment Therapy (ACT) is a psychotherapy with demonstrated efficacy for anxiety symptoms and disorders in the general population (Bluett et al., 2014) and, in a growing body of literature, for a variety of psychosocial and behavioral outcomes in cancer survivors (Mathew et al., 2020). A major strength of the ACT model (Hayes et al., 2012) is that it clearly specifies the six core theorized psychological processes that it targets: active acceptance (to counteract avoidance), cognitive defusion, present-moment awareness, self-ascontext, identification of values, and committed action (committing to behave more consistently with one's values). However, methodological limitations have constrained the empirical study of these core theorized ACT target processes. The present study aims to address multiple methodological limitations of the ACT mediation literature, including which target processes are examined, how they are measured, and in what treatment setting they are targeted, within a population of anxious cancer survivors enrolled in an ACT randomized controlled trial (RCT).

Regarding extant methodological constraints, first, psychometric concerns have arisen about several of the most widely used measures of the core theorized ACT target processes including in the domains of experiential avoidance (Tyndall et al., 2019; Wolgast, 2014) and values-aligned behavior (Barrett et al., 2019; Reilly et al., 2019). It is possible that previous studies' failure to detect movement on these variables results from the use of insufficiently sensitive or construct-specific instruments (Benoy et al., 2019). Second, studies examining mechanisms of ACT have naturally focused on the six core theorized ACT target processes (Stockton et al., 2019). Such studies typically have not examined plausible broader target 
processes of change as comparators, though two recent studies have examined one such target process, self-compassion, as a mediator of ACT (Ong, Barney et al., 2019; Ritzert et al., 2020). Third, the behavioral intervention literature has often examined mediation in the context of efficacy trials with rigorously trained, closely supervised, university-based therapists (often doctoral students or psychologists). Such studies offer, with regard to the therapists, limited approximations of real-world providers and settings that many consumers of psychological interventions encounter, wherein clinicians (often Master's level) may have little training and little or no ongoing supervision in specific behavioral intervention modalities such as ACT. It is critical to evaluate how evidence-based psychotherapies work in real-world contexts and whether they continue to uphold core theorized mechanisms in less controlled environments.

To better understand ACT's mechanisms, the cumulative evidence points toward several potential advances. First, experiential avoidance, a core theorized ACT target process, should be measured using instruments that do not conflate experiential avoidance with negative affect and distress, a criticism of the most widely used ACT process measure (Tyndall et al., 2019; Wolgast, 2014), the Acceptance and Action Questionnaire-II (AAQ-II; Bond et al., 2011). In addition, if the AAQ-II is used, then versions that probe experiential avoidance in specific relevant contexts may yield more accurate estimates of behavior (Ong et al., 2020). Second, given psychometric problems with other common measures of core theorized ACT target processes, such as measures of values-aligned behavior (Reilly et al., 2019), researchers should consider using multiple instruments of the same target process, probing the construct in different ways. Third, mediation studies should examine both core theorized ACT and more general psychotherapy target processes (e.g., Treanor et al., 2011). Finally, target processes require examination in real-world settings. 
The present study applies these methodological recommendations and advances in a mediation analysis of the Valued Living RCT (Arch et al., 2021). This RCT tested group-based ACT vs. minimally-enhanced usual care (MEUC) for anxious adults who recently completed cancer treatment and were transitioning into cancer survivorship, a challenging period wherein some cancer survivors experience a new onset or continuation of clinically significant anxiety disorders and related symptoms (e.g., Arch et al., 2020). The community oncology clinic setting where this study was conducted reflects the type of real-world setting where most U.S. cancer patients receive treatment (Petrelli, 2010) and thus would likely encounter cancer-specific supportive interventions. Consistent with the care model in this setting, the intervention was delivered by onsite oncology clinical social workers. The interventionists showed impressive adherence to the manualized intervention content (Arch et al., 2021) despite most of them having minimal or no prior ACT experience and limited study-specific training (reading sections of a book and completing a multi-day training for the study) and supervision (provided only during the first four of 12 cohorts of the RCT).

Within this context, we examined two core theorized ACT target processes: experiential avoidance and values-aligned behavior. Given their status within the theoretical (e.g., Hayes et al., 2012) and empirical (Stockton et al., 2019) literature as core processes targeted by ACT, as well as evidence implicating experiential avoidance (see review by Hayes-Skelton \& Eustis, 2020) and values (e.g., Gloster et al., 2017; Michelson et al., 2011) in the maintenance and treatment of anxiety, we hypothesized that improvement in each of these target processes would independently mediate ACT's effects on improvement in anxiety-related outcomes in the Valued Living RCT. We used two instruments per target process and followed recommendations in the literature to use a more psychometrically sound measure of experiential avoidance as well as a 
context-specific measure (Ong, Lee et al., 2019). Both of these processes were emphasized during the Valued Living intervention sessions using various experiential exercises as well as in assigned weekly home practice exercises.

We also examined two broader, ACT-consistent target processes, self-compassion and emotional approach coping. These two processes were examined as mediators because they: 1) were targeted by the Valued Living intervention; 2) are especially relevant in treatment of cancer survivorship-related anxiety; and 3) are consistent with, but not part of, the ACT theoretical model (Hayes et al., 2012).

Self-compassion is consistent with ACT's emphasis on flexible perspective taking and psychological flexibility, and is targeted in many mindfulness- and acceptance-based psychotherapies (A. C. Wilson et al., 2019), though is not one of the core ACT target processes specified by Hayes and colleagues (2012). Low self-compassion is associated with higher anxiety in chronic health populations, including cancer patients (see review by Hughes et al., 2021), further highlighting self-compassion as an intriguing target process in the current study sample of cancer survivors. Operationalizing the components of self-compassion conceptualized by Neff (2003), the Valued Living intervention facilitator manual instructed interventionists to promote self-kindness as a response to making mistakes and to emphasize common humanity by drawing participants' attention to their shared experiences. We thus hypothesized that increases in self-compassion would mediate ACT's effects on improvement in anxiety-related outcomes.

Emotional approach coping, defined as coping with stressors by actively acknowledging, processing, and expressing one's emotions (Stanton, Kirk, et al., 2000), also likely is promoted in many psychotherapeutic modalities and has been shown to predict psychological well-being in breast cancer survivors (Stanton, Danoff-Burg, et al., 2000). Given these previous findings, we 
hypothesized that increases in emotional approach coping would mediate ACT's effects on improvement in anxiety-related outcomes. Valued Living interventionists were instructed to promote emotional approach coping by encouraging and reinforcing participants' self-reflection and sharing their experiences and emotions related to cancer survivorship with the group.

The aims of the present study were to examine whether these core theorized ACT and broader ACT-consistent target processes showed greater improvement in the ACT condition as compared to MEUC, and whether longitudinal improvements in the target processes were associated with, and statistically mediated, the primary outcome of the trial (general anxiety symptoms) and related anxiety outcomes (fear of cancer recurrence and cancer trauma-related symptoms).

\section{Methods}

\section{Participants}

To be eligible for the Valued Living RCT, participants were required to: 1) have completed primary cancer treatment within 1.5-24 months of study enrollment; 2) have no current evidence of cancer for solid tumor cancers, or be in remission or asymptomatic after initial treatment for hematologic malignancies; 3) self-report elevated anxiety about cancer and elevated general anxiety and/or depressive symptoms on multiple screeners ${ }^{1}$; 4) be proficient in English. Participants $(N=134)$ were on average 56.24 years old $(S D=11.58)$, and the majority identified as female (88.10\%) and White, non-Latinx (86.60\%); additional demographic information is provided in Supplemental Table 1.

\section{Design}

Participants were randomly assigned 1:1 to group-format ACT delivered by oncology

\footnotetext{
${ }^{1}$ All participants had to report current anxiety about cancer or cancer survivorship; $97 \%$ also screened positive for anxiety symptoms in daily life and 57\% screened positive for depressive symptoms in daily life (Arch et al, 2021).
} 
clinical social workers in a community oncology setting or to MEUC. The current analysis focuses on the assessments at Pre-intervention (Pre, prior to randomization) and Postintervention (Post).

\section{Measures}

All measures were administered in Qualtrics (Provo, UT). Summary statistics for the baseline scores on each measure, and comparisons of baseline score differences between ACT and MEUC, are provided in Supplemental Table 1. For variables that showed a significant difference on baseline scores between ACT and MEUC, we computed a Cohen's $d$ effect size and obtained a percentile bootstrapped 95\% confidence intervals (CI) from 10,000 nonparametric bootstrapped samples.

\section{Outcomes}

We examined mediation for the anxiety-related outcome measures, each of which showed significant or marginally significant condition differences in change from Pre to 6-month followup in the main outcome analyses (Arch et al., 2021). Anxiety symptom outcomes were measured with the Hospital Anxiety and Depression Scale-Anxiety subscale (Pre $\alpha=.64$; Zigmond \& Snaith, 1983). Cancer-related trauma symptoms were measured with the Impact of Events ScaleRevised total score (IES-R, Pre $\alpha=.86$; Weiss \& Marmar, 1997), and fear of cancer recurrence was measured with the Concerns About Recurrence Scale-Overall Fears score (CARS, Pre $\alpha=$ .85; Vickberg, 2003), replacing "breast cancer" with "cancer” (Arch \& Mitchell, 2016).

\section{Core Theorized ACT Target Processes}

Experiential Avoidance. General experiential avoidance was measured with the 15 -item Brief Experiential Avoidance Questionnaire (BEAQ, Pre $\alpha=.79$; Gámez et al., 2014), which has good psychometric properties (e.g., Gámez et al., 2014; Tyndall et al., 2019). Cancer-specific 
experiential avoidance was measured with the 16-item Acceptance and Action

Questionnaire-Cancer scale (AAQc, Pre $\alpha=.84$; Arch \& Mitchell, 2016), a cancer-specific adaptation of the AAQ-II demonstrated to change following group ACT for cancer survivors in a single-arm trial (Arch \& Mitchell, 2016). Compared to the AAQ-II, the AAQc has more behaviorally-focused items and probes experiential avoidance in the context of cancer. Lower scores on the BEAQ and AAQc indicate lower experiential avoidance. ACT participants scored higher on the AAQc $(p=.047, d[95 \% \mathrm{CI}]=0.35[0.00,0.73]$; Supplemental Table 1) than MEUC participants at Pre.

Values-aligned Behavior. Values-aligned behavior was measured with two instruments that use different measurement approaches. The Bull's-Eye Values Survey (henceforth Bullseye, Pre $\alpha=$.69; Lundgren et al., 2012) measures values-consistent behavior with a visual bullseye diagram. The Valued Living Questionnaire (VLQ, Pre composite $\alpha=.84$; K. G. Wilson et al., 2010) asks respondents to rate the a) personal importance of, and b) behavioral consistency with, values in each of ten life domains. A composite score is computed by multiplying importance and consistency scores for each domain, and the summed composite score is analyzed. In a systematic review of values-aligned behavior measures, Reilly and colleagues (2019) found that the Bullseye and VLQ showed sensitivity to intervention in some studies, though empirical support for their psychometric qualities, such as internal consistency and readability, was mixed or not assessed. More values-consistent behavior is indicated by lower Bullseye and higher VLQ scores.

\section{Broader ACT-Consistent Target Processes}

Self-compassion was measured with the 12-item Self-Compassion Scale-Short Form (SCS, Pre $\alpha=.87$; Raes et al., 2011), which has shown sensitivity to change following ACT 
(Ong, Barney et al., 2019; Ritzert et al., 2020). Cancer-related emotional approach coping was measured with the emotional approach coping scale 8-item mean score (Pre $\alpha=.93$; Stanton, Kirk, et al., 2000). This scale has not been used in prior ACT research to our knowledge but is consistent with ACT's (and many other psychotherapies') emphasis on approaching rather than avoiding emotions. Higher scores indicate greater self-compassion or emotional approach coping.

\section{Missing Data}

Due to an administrative error, the BEAQ and Bullseye were not administered to the first half of enrolled participants at Pre.

\section{Analytic Approach}

We examined mediation effects using structural equation models. The pre-registered plan (https://osf.io/akwpf) was to examine these phenomena using latent growth curves; however, these models failed to converge consistently, likely due to a lack of between-participant random variance in target process slopes.

We therefore proceeded to examine mediation over the Pre-Post interval, wherein change on target process and outcome variables was most likely to occur (e.g., Goldsmith et al, 2018), by looking at change in target processes and outcomes from Pre to Post. We used the latent change score framework proposed by Valente and MacKinnon (2017), focusing initially on the ANCOVA-equivalent models recommended by Valente and MacKinnon on empirical and theoretical grounds, and the difference score models because they aligned with common approaches to mediation (e.g., Judd et al., 2001; Montoya and Hayes, 2017). Because the ANCOVA-equivalent mediation models fit the data significantly better than the difference score models for all mediator-outcome combinations (nested model comparison $\Delta \chi^{2} \mathrm{~s}[d f=4] \geq 34.937$, 
$p \mathrm{~s}<.001)$, we proceeded to estimate ANCOVA-equivalent models as the final models.

The ANCOVA-equivalent model (see Figure 1b), like the difference score model (e.g., Judd et al., 2001), estimates the effect of condition assignment on change over two timepoints in a mediating variable ( $a$ path), and the effect of change over two timepoints in a mediating variable on change over two timepoints in an outcome variable ( $b$ path). We assessed the evidence for mediation by examining the statistical significance and confidence intervals of the indirect effect $a b$ using bootstrapped standard errors (e.g., Preacher \& Hayes, 2004). The ANCOVA-equivalent model adjusts for the effects of pre-intervention scores of the mediator and outcome variables on post-intervention scores of the mediator and outcome variables, whereas those paths are implicitly assumed to be equal to zero in the difference score model (Valente and MacKinnon, 2017). We computed unmediated versions of the ANCOVA-equivalent models (Figure 1a) to derive c paths for the three outcome variables. When an outcome was significantly mediated in these individual (single) mediation models by more than one target process variable, we then evaluated the significant individual mediators together in a multiple mediation model for that outcome (Figure 2). No additional covariates were included in the unmediated or mediated models. Statistical significance was defined as $p<.05$.

[Figure 1 approximately here]

The latent change score models were estimated in R (Rosseel, 2012) with bootstrapping (10,000 nonparametric samples) to compute standard errors. The mediation model syntax is available online (https://osf.io/xfm8b/). As pre-registered, we used full information maximum likelihood estimation. Because the modeling approach shifted from the pre-registered plan, we reduced the number of examined models from what was pre-registered in order to reduce Type I error risk. Specifically, we analyzed only the anxiety-related outcomes, given the trial's focus on 
anxious cancer survivors and the established theoretical and empirical links between anxiety reduction and experiential avoidance (Hayes-Skelton \& Eustis, 2020), values-aligned behavior (Barrett et al., 2019), and self-compassion (A. C. Wilson et al., 2019). Additionally, we analyzed the emotional approach coping total score rather than its subscales and refrained from conducting other proposed secondary analyses. Because there were no significant cohort-clustering effects in the main RCT outcomes (Arch et al., 2021), cohort was not modeled in the present study.

[Figure 2 approximately here]

To evaluate the effect size of any observed marginal or significant mediated effects, we examined $a b / s_{Y}$ for the single mediator models, and the total mediated effect $\left(a_{1} b_{1}+a_{2} b_{2}\right) / s_{Y}$ (e.g., MacKinnon, 2007) for the multiple mediation models, and computed 95\% CIs for the effect size using percentile bootstrap estimates. We used $a b / s_{Y}$ and percentile bootstrapped estimates because in simulation studies by Miočević and colleagues (2018) these statistics outperformed other commonly-used mediation effect size estimation techniques. Estimates of $a b / s_{Y}$ are equivalent to a Cohen's $d$, but reflect only the indirect effect (i.e., $a b$ ) in standardized units. These values will necessarily be smaller than the overall effect size of treatment on a given outcome. Thus, canonical Cohen's $d$ cutoffs of large, medium, and small effect sizes are inappropriate in interpreting the magnitude of effect sizes in this mediation context (Miočević et al., 2018).

Since the latent change score models do not yield readily interpretable estimates of average Pre-Post change across the sample, we computed means and standard errors of participants Pre-Post change scores and tested whether they were significantly different from zero with a one-sample $t$-test (Supplemental Table 2). 


\section{Results}

\section{Change in Target Processes}

There was significant overall improvement in all target processes (Supplemental Table 2). ACT and MEUC participants did not differ significantly in Pre-Post change on the two experiential avoidance measures (Table $1 a$ paths). Comparing the two values-aligned behavior measures, ACT improved more on the Bullseye, but not on the VLQ. By contrast, ACT led to significantly greater improvement than MEUC on the self-compassion and emotional approach coping measures.

[Table 1 approximately here]

\section{Change in Outcome Variables}

Overall across condition, each of the three anxiety-related outcomes showed significant improvement from Pre to Post (Supplemental Table 2). In the $c$ paths of unmediated models (e.g., Figure 1a), ACT participants evidenced significantly greater Pre-Post improvement in cancer-related trauma symptoms $(c$ estimate $=-0.31, \mathrm{SE}=0.10, p=.002)$ and fear of cancer recurrence $(c$ estimate $=-0.65, \mathrm{SE}=0.17, p<.001)$, but not general anxiety symptoms $(c$ estimate $=-0.22, \mathrm{SE}=0.51, p=.663)$. Given these findings, in discussing residual intervention effects ( $c^{\prime}$ paths) below, we focused on the trauma symptom and fear of cancer recurrence outcomes to examine mediation.

\section{Target Process-Outcome Associations and Single Mediation}

\section{Core ACT-Theorized Target Processes}

Experiential Avoidance. As illustrated in Table 1, Pre-Post improvement in both measures of experiential avoidance (AAQc and BEAQ) was associated with improvement in cancer-related trauma symptoms, fear of cancer recurrence, and general anxiety symptom 
outcomes after controlling for condition ( $b$ paths). However, the mediation effects of both the BEAQ and AAQc on these outcomes were nonsignificant $(a b)$.

Values-aligned Behavior. Improvement in Bullseye scores was associated with improvement in fear of cancer recurrence, cancer-related trauma symptoms, and general anxiety symptoms (Table $1 b$ paths). Change in Bullseye approached significance as a mediator of improvement in fear of cancer recurrence $\left(p=.088 ; a b / s_{Y}[95 \% \mathrm{CI}]=-0.18[-0.46,-0.01]\right)$ and general anxiety $\left(p=.071 ; a b / s_{Y}[95 \% \mathrm{CI}]=[-0.45,0.01]\right)$ outcomes, but not cancer-related trauma outcomes. Residual intervention effects remained significant for cancer-related trauma symptoms and fear of cancer recurrence ( $c^{\prime}$ paths), suggesting partial mediation at the marginal level. VLQ improvement was only marginally associated with general anxiety symptom improvement ( $b$ path; $p=.085$ ) and not associated with improvement on other outcomes, and mediation was not supported.

\section{Broader ACT-Consistent Target Processes}

Self-compassion. Greater increases in self-compassion were associated with greater improvements in cancer-related trauma symptoms, fear of cancer recurrence, and general anxiety symptoms (Table $1 b$ paths). Self-compassion was a significant mediator of cancer-related trauma symptoms $\left(p=.037 ; \mathrm{ab} / s_{Y}[95 \% \mathrm{CI}]=-0.26[-0.53,-0.04] ;\right.$ see also Figure $\left.1 \mathrm{~b}\right)$ and a marginal mediator of fear of cancer recurrence $\left(p=.081 ; \mathrm{ab} / s_{Y}[95 \% \mathrm{CI}]=-0.16[-0.37,0.01]\right)$, and general anxiety symptoms $\left(p=.079 ; a b / s_{Y}[95 \% \mathrm{CI}]=-0.18[-0.42,0.00]\right)$. Because the residual intervention effects remained significant for cancer-related trauma symptoms and fear of cancer recurrence ( $c^{\prime}$ paths), self-compassion served as a partial mediator.

Emotional Approach Coping. Greater increases in emotional approach coping were associated with significantly greater improvements in cancer-related trauma symptoms and fear 
of cancer recurrence, but only marginally so for general anxiety symptoms (Table $1 b$ paths). The $a b$ coefficients were significant for cancer-related trauma symptoms $\left(p=.022 ; \mathrm{ab} / s_{Y}[95 \% \mathrm{CI}]=\right.$ $-0.23[-0.46,-0.07])$ and marginal for fear of cancer recurrence $\left(p=.065 ; \mathrm{ab} / \mathrm{s}_{Y}[95 \% \mathrm{CI}]=-0.16\right.$ $[-0.37,-0.02])$ and general anxiety outcomes $\left(p=.086 ; \mathrm{ab} / \mathrm{s}_{Y}[95 \% \mathrm{CI}]=-0.13[-0.29,0.02]\right)$; thus, emotional approach coping was established as a significant or marginal mediator of the intervention effect on these outcomes. Because residual intervention effects were significant $\left(c^{\prime}\right.$ paths) for cancer-related trauma symptoms and general anxiety outcomes, partial mediation was indicated.

\section{Multiple Mediation of Cancer-Related Trauma Symptoms}

Given that statistically significant mediators were identified only for the cancer-related trauma-symptom variable, we examined multiple mediation of trauma-symptom change by change in self-compassion and emotional approach coping scores. ${ }^{2}$ A path diagram of this model is provided in Figure 2.

[Table 2 approximately here]

In the multiple mediation model, both self-compassion and emotional approach coping retained significant $a$ and $b$ paths (Table 2). The self-compassion indirect effect $(a b)$ was significant $(p=.030)$, whereas the emotional approach coping indirect effect was marginal $(p=$ .089). The overall indirect effect (reflecting the summed effect of both mediators) was significant $\left(p=.008 ;\left[a_{1} b_{1}+a_{2} b_{2}\right] / s_{Y}[95 \% \mathrm{CI}]=-0.42[-0.78,-0.15]\right)$, and the residual intervention effect (c') path was nonsignificant, suggesting full mediation.

\section{Discussion}

This study examined longitudinal change and mediation effects of core theorized ACT

\footnotetext{
${ }^{2}$ The model-estimated correlation of latent self-compassion Pre-Post scores and latent emotional approach coping Pre-Post scores was $r=0.23$; thus, problems with collinearity of the mediators are unlikely.
} 
and broader ACT-consistent target processes in a community oncology clinic-based RCT of ACT versus MEUC for anxious cancer survivors. Regarding condition differences, ACT participants showed significantly greater improvement than MEUC participants on one measure of values-aligned behavior but not on either measure of experiential avoidance. ACT participants also improved more than MEUC on two broader ACT-consistent target processes, selfcompassion and emotional approach coping. Regarding mediation effects, self-compassion and emotional approach coping each were significant partial mediators of cancer-related trauma symptoms. In a multiple mediation model, each of these mediators made a unique and additive contribution to predicting cancer-related trauma symptoms. In addition, self-compassion, emotional approach coping, and values-aligned behavior were each marginally significant partial mediators of fear of cancer recurrence and general anxiety symptoms. In summary, both of the broader ACT-consistent target processes were significant or marginal partial mediators in the present community oncology-implemented ACT intervention (and together, fully mediated change in cancer-related trauma symptoms in a multiple mediation model), but only one of the four measures of core ACT target processes was even a marginally significant partial mediator.

The positive finding that self-compassion served as a significant or marginal mediator of anxiety-related outcomes for anxious cancer survivors adds to the small but growing literature evaluating self-compassion as a mediator of ACT's effects on diverse outcomes. A trial of 10 sessions of ACT versus waitlist control for clinical perfectionism showed that self-compassion increased more with ACT, and that increased self-compassion during the trial was associated with a reduction in perfectionism from post-intervention to follow-up (though $a b$ was not reported; Ong, Barney et al., 2019). By contrast, self-compassion did not mediate the effects of an ACT-based bibliotherapy intervention on anxiety symptom severity, relative to a waitlist 
control condition (Ritzert et al., 2020). The present study adds to this literature by demonstrating that within a multi-session group ACT intervention for cancer survivors delivered in the community, self-compassion had significant or trending mediating effects on anxiety-related outcomes.

The emotional approach coping findings align with evidence that higher levels of emotional approach coping are associated with decreased psychological distress among cancer survivors (Stanton, Danoff-Burg, et al., 2000). Notably, the present study is, to our knowledge, the first to examine emotional approach coping as a mediator of ACT. Our findings highlight the overlap between ACT's emphasis on acceptance of internal experience and the broader literature on opening up to emotions as an effective coping strategy for many individuals in stressful contexts such as cancer (Stanton, Danoff-Burg, et al., 2000).

The findings from the multiple mediation model and effect size estimates help to contextualize the relative contributions that these mediators make to the overall effect of the ACT intervention. Interestingly, self-compassion remained a statistically significant unique mediator in the multiple mediation model, whereas emotional approach coping was only a marginally significant mediator in that model. However, the total mediated effect size in the multiple mediation model was larger than those in the single mediation models, indicating that the combined effects of self-compassion and emotional approach coping were additive to some degree rather than overlapping. Indeed, the residual treatment effect was no longer statistically significant when both mediators were included in the model, suggesting full mediation. The point estimate of the total mediated effect size implied that assignment to the ACT condition was associated with approximately $42 \%$ of a standard deviation more improvement in trauma symptoms due to improvement in self-compassion and emotional approach coping. While it is 
tempting to apply the canonical cutoffs for Cohen's $d$ effect size magnitudes to those observed here, Miočević and colleagues (2018) caution that mediated effect sizes will necessarily be smaller than overall intervention effect sizes to which those standards are typically applied, and that further research is needed to establish what would constitute a 'large' or 'small' mediated effect size in a given research domain.

The evidence for valued-aligned behavior as a mediator of ACT on anxiety-related outcomes was mixed. The Bullseye instrument was a marginally significant partial mediator of change in fear of cancer recurrence and general anxiety symptoms. Unfortunately, due to an administrative error, approximately half the participants did not complete the Bullseye measure. Although we were able to use all available data by virtue of full information maximum likelihood estimation, the smaller number of observations likely weakened the precision with which we were able to estimate mediation with the Bullseye measure. In contrast to the Bullseye, VLQ scores did not improve more with ACT intervention, mediate anxiety-related outcomes, or even consistently track changes in anxiety-related outcomes. It is notable that two instruments that are intended to measure the same construct yielded divergent findings; further study is needed to determine whether the VLQ and Bullseye, two commonly-used measures of valuesaligned behavior (Reilly et al., 2019), do not converge.

Experiential avoidance, as assessed with both cancer-specific (AAQc) and general (BEAQ) measures, did not improve significantly more with ACT intervention. This finding is unexpected given that numerous studies (reviewed by Stockton et al., 2019), including studies with cancer populations (e.g., Hawkes et al., 2013), have shown that experiential avoidance decreases more with ACT and mediates its effects on anxiety and other psychosocial outcomes. The negative association between cancer-related avoidance and anxiety was also evident in the 
single-arm pilot study predecessor to the current RCT (Arch \& Mitchell, 2016), which found that AAQc score improvement from pre- to post-intervention was marginally associated with subsequent anxiety improvement. This earlier finding was analogous to the significant $b$ paths observed in the present study. The present study's finding of equivalent experiential avoidance improvement between conditions (i.e., null $a$ paths) was surprising, and could have occurred due to methodological issues that may have masked true positive effects. First, the considerable missing data at Pre on the BEAQ in the present study may have led to a lack of power to detect intervention effects. In addition, the AAQ-II (which provided many of the item stems for the AAQc) has previously shown failure to capture intervention effects (Benoy et al., 2019). It is possible that the AAQc is inconsistently sensitive to treatment effects, and thus failed to capture actual experiential avoidance change in the Valued Living RCT. Overall, it is striking that both measures of experiential avoidance did not change significantly more with ACT, a finding worthy of future investigation.

\section{Study Strengths and Limitations}

A key strength of this study is its examination of target processes that are strongly theoretically linked to ACT, as well as processes that are relevant to most contemporary psychotherapies, within an RCT conducted in a real-world clinical context. Real-world environments are essential testing grounds for treatment mechanisms, as non-researcher clinicians provide the vast majority of supportive and mental health care. For example, oncology clinical social workers (who led the current ACT intervention) represent the majority of psychooncology care providers (Deshields et al, 2016), yet only a minority of social work training programs require training in evidence-based psychotherapies (Weissman et al., 2006). The present study highlights that, in such real-world contexts, evidence-based interventions may not 
operate according to the theoretically expected mechanisms, a finding that should be further explored in both oncology and more general patient populations and settings. Another key strength of this study was its measurement of values-aligned behavior and experiential avoidance with two instruments each, and its use of a more psychometrically sound measure (i.e., the BEAQ; Tyndall et al., 2019) and a context-specific measure (i.e., the AAQc; Ong et al., 2020) of experiential avoidance.

With regard to limitations, first, the considerable missing data on the BEAQ and Bullseye warrant cautious interpretation of the findings on these measures. As discussed above, these missing data likely impeded the precision of the Bullseye mediation effect estimates. However, we had approximately 60 observations of Pre-Post scores for each of these variables, a sample size that is comparable to numerous other ACT RCT mediation studies to date (Stockton et al., 2019), including ones that have successfully demonstrated mediation (e.g., Arch et al., 2012). Furthermore, the BEAQ and Bullseye had counterpart instruments of the same theorized ACT constructs, the AAQc and VLQ, respectively, which did not have significant missing data. Thus, we were able to assess mediation of each core theorized ACT target process with one variable that had robust data. As noted above, the present study is unique in this regard; most ACT mediation studies do not include multiple instruments per target process.

A second limitation of this trial emerged from the failure of randomization to yield equivalent scores on the AAQc, which presents a potential threat to internal validity. The observed baseline condition difference was unexpected and could have been a statistically spurious finding. However, we cannot rule out that the conditions may have actually differed at baseline to some degree.

Next, the current study examined contemporaneous mediation (i.e., change in Pre-Post 
mediator scores predicting change in Pre-Post outcome scores). Although the logic of mediation implies that change in the mediator should precede change in the outcome, we refrained from examining lagged mediation given that the timing of the assessments in this trial was spaced by a month or more, which was likely a bigger window than that in which the lagged effect of a mediator on an outcome occurs in a psychotherapy context. Goldsmith and colleagues (2018) argued that this timing issue is a reason why many other trials of psychotherapeutic interventions have found contemporaneous mediation models to fit better than lagged mediation models. As they and others (e.g., Hofmann et al., 2020) note, greater attention is needed in future studies to capture the time course of lagged effects of target process variables on outcomes. Likely such trial designs would require intensive data collection, which was outside the scope of the current RCT, given our goal of testing the intervention in a community setting where intensive measurement poses significant burden and does not reflect clinical practice. Following the example of the recent Association for Contextual Behavior Science task force report (Hayes et al., 2021), we highlight such data collection as an important future direction for ACT mediation research and psychological intervention research generally, to better characterize the time course and direction (or even recursivity) of effects between target processes and outcomes (e.g., Hofmann et al., 2020). The present research demonstrates the potential importance of measuring and modeling broader ACT-consistent target processes in future research that uses more intensive sampling.

Finally, though we have focused on mediation of anxiety-related outcomes given the strong evidence for ACT as a treatment for anxiety disorders (e.g., Bluett et al., 2014) and the role of anxiety symptoms as the primary outcome in the present RCT (Arch et al, 2021), we have not examined mediation of other outcomes (e.g., sense of life meaning or well-being) that are 
aligned with ACT's aim to promote adaptive functioning and flourishing. As noted, we had originally planned to examine additional outcomes, but elected to focus on the trial's primary outcome and related anxiety outcomes to reduce risk of Type I error upon being required to shift from the pre-registered analytic plan. It will be important in future ACT studies to examine mediation of outcomes such as quality of life or sense of life meaning, in addition to mediation of symptom outcomes.

\section{Conclusion}

We have examined core theorized ACT and broader ACT-consistent target processes in an ACT RCT for anxious cancer survivors embedded within community oncology care clinics. Values-aligned behavior as measured by one instrument (but not another) was a marginal partial mediator of fear of cancer recurrence and general anxiety symptom improvement. The two broader ACT-consistent target processes (self-compassion and emotional approach coping) were significant partial mediators of cancer-related trauma symptoms and marginally significant partial mediators of fear of cancer recurrence and general anxiety symptom improvement, and together, in a multiple mediation model, fully mediated change on cancer-related trauma symptoms. By contrast, neither general nor cancer-related experiential avoidance improved more in the ACT condition, nor they did not mediate improvement in any anxiety-related outcomes. The current study thus highlights the possibility that, for real-world implementation of ACT, target processes consistent with ACT yet shared among many psychotherapies may strongly contribute to ACT's overall beneficial effects. 


\section{References}

Arch, J. J., Genung, S. R., Ferris, M. C., Kirk, A., Slivjak, E. T., Fishbein, J. N., Schneider, R. L., \& Stanton, A. L. (2020). Presence and predictors of anxiety disorder onset following cancer diagnosis among anxious cancer survivors. Supportive Care in Cancer, 28(9), 4425-4433. https://doi.org/10.1007/s00520-020-05297-0

Arch, J. J., \& Mitchell, J. L. (2016). An Acceptance and Commitment Therapy (ACT) group intervention for cancer survivors experiencing anxiety at re-entry. Psycho-Oncology, 25(5), 610-615. https://doi.org/10.1002/pon.3890

Arch, J. J., Mitchell, J. L., Genung, S. R., Judd, C. M., Andorsky, D. J., Bricker, J. B., \& Stanton, A. L. (2021). Randomized trial of an Acceptance and Commitment Therapy intervention for anxious cancer survivors in community clinics: Outcomes and moderators. Journal of Clinical and Consulting Psychology, 89(4), 327-340. https://doi.org/10.1037/ccp0000630

Arch, J. J., Wolitzky-Taylor, K. B., Eifert, G. H., \& Craske, M. G. (2012). Longitudinal treatment mediation of traditional cognitive behavioral therapy and acceptance and commitment therapy for anxiety disorders. Behaviour Research and Therapy, 50(7-8), 469-478. https://doi.org/10.1016/j.brat.2012.04.007

Barrett, K., O’Connor, M., \& McHugh, L. (2019). A systematic review of values-based psychometric tools within Acceptance and Commitment Therapy (ACT). Psychological Record, 69(4), 457-485. https://doi.org/10.1007/s40732-019-00352-7

Benoy, C., Knitter, B., Schumann, I., Bader, K., Walter, M., \& Gloster, A. T. (2019). Treatment sensitivity: Its importance in the measurement of psychological flexibility. Journal of Contextual Behavioral Science, 13, 121-125. https://doi.org/10.1016/j.jcbs.2019.07.005

Bluett, E. J., Homan, K. J., Morrison, K. L., Levin, M. E., \& Twohig, M. P. (2014). Acceptance 
and commitment therapy for anxiety and OCD spectrum disorders: An empirical review. Journal of Anxiety Disorders, 28(6), 612-624. https://doi.org/10.1016/j.janxdis.2014.06.008

Bond, F. W., Hayes, S. C., Baer, R. A., Carpenter, K. M., Guenole, N., Orcutt, H. K., Waltz, T., \& Zettle, R. D. (2011). Preliminary psychometric properties of the Acceptance and Action Questionnaire-II: A revised measure of psychological inflexibility and experiential avoidance. Behavior Therapy, 42(4), 676-688. https://doi.org/10.1016/j.beth.2011.03.007

Deshields, T., Kracen, A., Nanna, S., \& Kimbro, L. (2016). Psychosocial staffing at National Comprehensive Cancer Network member institutions: Data from leading cancer centers. Psycho-Oncology, 25(2), 164-169. https://doi.org/10.1002/pon.3826

Gámez, W., Chmielewski, M., Kotov, R., Ruggero, C., Suzuki, N., \& Watson, D. (2014). The Brief Experiential Avoidance Questionnaire: Development and initial validation. Psychological Assessment, 26(1), 35-45. https://doi.org/10.1037/a0034473

Gloster, A. T., Klotsche, J., Ciarrochi, J., Eifert, G., Sonntag, R., Wittchen, H.-U., \& Hoyer, J. (2017). Increasing valued behaviors precedes reduction in suffering: Findings from a randomized controlled trial using ACT. Behaviour Research and Therapy, 91, 64-71. https://doi.org/10.1016/j.brat.2017.01.013

Goldsmith, K. A., MacKinnon, D. P., Chalder, T., White, P. D., Sharpe, M., \& Pickles, A. (2018). Tutorial: The practical application of longitudinal structural equation mediation models in clinical trials. Psychological Methods, 23(2), 191-207. https://doi.org/10.1037/met0000154

Hawkes, A. L., Chambers, S. K., Pakenham, K. I., Patrao, T. A., Baade, P. D., Lynch, B. M., 
Aitken, J. F., Meng, X., \& Courneya, K. S. (2013). Effects of a telephone-delivered multiple health behavior change intervention (CanChange) on health and behavioral outcomes in survivors of colorectal cancer: A randomized controlled trial. Journal of Clinical Oncology, 31(18), 2313-2321. https://doi.org/10.1200/JCO.2012.45.5873

Hayes, S. C., Strosahl, K. D., \& Wilson, K. G. (2012). Acceptance and Commitment Therapy (2nd ed.). Guilford Press.

Hayes, S. C., Merwin, R. M., McHugh, L., Sandoz, E. K., A-Tjak, J. G. L., Ruiz, F. J., BarnesHolmes, D., Bricker, J. B., Ciarrochi, J., Dixon, M. R., Fung, K. P.-L., Gloster, A. T., Gobin, R. L., Gould, E. R., Hofmann, S. G., Kasujja, R., Karekla, M., Luciano, C., \& McCracken, L. M. (2021). Report of the ACBS Task Force on the strategies and tactics of contextual behavioral science research. Journal of Contextual Behavioral Science, 20, 172-183. https://doi.org/10.1016/j.jcbs.2021.03.007

Hayes-Skelton, S. A., \& Eustis, E. H. (2020). Experiential avoidance. In J. Abramowitz \& S. M. Blakey (Eds.), Clinical handbook of fear and anxiety: Maintenance processes and treatment mechanisms. American Psychological Association.

Hofmann, S. G., Curtiss, J. E., \& Hayes, S. C. (2020). Beyond linear mediation: Toward a dynamic network approach to study treatment processes. Clinical Psychology Review, 76, 101824. https://doi.org/10.1016/j.cpr.2020.101824

Hughes, M., Brown, S. L., Campbell, S., Dandy, S., \& Cherry, M. G. (2021). Self-compassion and anxiety and depression in chronic physical illness populations: A systematic review. Mindfulness, 12(7), 1597-1610. https://doi.org/10.1007/s12671-021-01602-y

Judd, C. M., Kenny, D. A., \& McClelland, G. H. (2001). Estimating and testing mediation and moderation in within-subject designs. Psychological Methods, 6(2), 115-134. 
https://doi.org./10.1037/1082-989X.6.2.115

Lundgren, T., Luoma, J. B., Dahl, J., Strosahl, K., \& Melin, L. (2012). The Bull's-Eye Values Survey: A psychometric evaluation. Cognitive and Behavioral Practice, 19(4), 518-526. https://doi.org/10.1016/j.cbpra.2012.01.004

MacKinnon, D. P. (2007). Single mediator model details. In Introduction to statistical mediation analysis (pp. 79-102). Erlbaum.

Mathew, A., Doorenbos, A. Z., Jang, M. K., \& Hershberger, P. E. (2020). Acceptance and commitment therapy in adult cancer survivors: A systematic review and conceptual model. Journal of Cancer Survivorship. https://doi.org/10.1007/s11764-020-00938-z

Michelson, S. E., Lee, J. K., Orsillo, S. M., \& Roemer, L. (2011). The role of values-consistent behavior in generalized anxiety disorder. Depression and Anxiety, 28(5), 358-366. https://doi.org/10.1002/da.20793

Miočević, M., O’Rourke, H. P., MacKinnon, D. P., \& Brown, H. C. (2018). Statistical properties of four effect-size measures for mediation models. Behavior Research Methods, 50(1), 285-301. https://doi.org/10.3758/s13428-017-0870-1

Montoya, A. K., \& Hayes, A. F. (2017). Two-condition within-participant statistical mediation analysis: A path-analytic framework. Psychological Methods, 22(1), 6-27. https://doi.org/10.1037/met0000086

Neff, K. (2003). Self-compassion: An alternative conceptualization of a healthy attitude toward oneself. Self and Identity, 2(2), 85-101. https://doi.org/10.1080/15298860309032

Ong, C. W., Barney, J. L., Barrett, T. S., Lee, E. B., Levin, M. E., \& Twohig, M. P. (2019). The role of psychological inflexibility and self-compassion in acceptance and commitment therapy for clinical perfectionism. Journal of Contextual Behavioral Science, 13, 7-16. 
https://doi.org/10.1016/j.jcbs.2019.06.005

Ong, C. W., Lee, E. B., Levin, M. E., \& Twohig, M. P. (2019). A review of AAQ variants and other context-specific measures of psychological flexibility. Journal of Contextual Behavioral Science, 12, 329-346. https://doi.org/10.1016/j.jcbs.2019.02.007

Ong, C. W., Pierce, B. G., Petersen, J. M., Barney, J. L., Fruge, J. E., Levin, M. E., \& Twohig, M. P. (2020). A psychometric comparison of psychological inflexibility measures: Discriminant validity and item performance. Journal of Contextual Behavioral Science, 18, 34-47. https://doi.org/10.1016/j.jcbs.2020.08.007

Petrelli, N. J. (2010). A community cancer center program: Getting to the next level. Journal of the American College of Surgeons, 210(3), 261-270. https://doi.org/10.1016/j.jamcollsurg.2009.11.015

Preacher, K. J., \& Hayes, A. F. (2004). SPSS and SAS procedures for estimating indirect effects in simple mediation models. Behavior Research Methods, Instruments, \& Computers, 36(4), 717-731. https://doi.org/10.3758/BF03206553

Raes, F., Pommier, E., Neff, K. D., \& Gucht, D. V. (2011). Construction and factorial validation of a short form of the Self-Compassion Scale. Clinical Psychology \& Psychotherapy, 18(3), 250-255. https://doi.org/10.1002/cpp.702

Reilly, E. D., Ritzert, T. R., Scoglio, A. A. J., Mote, J., Fukuda, S. D., Ahern, M. E., \& Kelly, M. M. (2019). A systematic review of values measures in acceptance and commitment therapy research. Journal of Contextual Behavioral Science, 12, 290-304. https://doi.org/10.1016/j.jcbs.2018.10.004

Ritzert, T. R., Berghoff, C. R., Tifft, E. D., \& Forsyth, J. P. (2020). Evaluating ACT processes in relation to outcome in self-help treatment for anxiety-related problems. Behavior 
Modification, 44(6), 865-890. https://doi.org/10.1177/0145445519855616

Rosseel, Y. (2012). lavaan: An R package for structural equation modeling. Journal of Statistical Software, 48(1), 1-36. https://doi.org/10.18637/jss.v048.i02

Stanton, A. L., Danoff-Burg, S., Cameron, C. L., Bishop, M., Collins, C. A., Kirk, S. B., Sworowski, L. A., \& Twillman, R. (2000). Emotionally expressive coping predicts psychological and physical adjustment to breast cancer. Journal of Consulting and Clinical Psychology, 68(5), 875-882. https://doi.org/10.1037/0022-006X.68.5.875

Stanton, A. L., Kirk, S. B., Cameron, C. L., \& Danoff-Burg, S. (2000). Coping through emotional approach: Scale construction and validation. Journal of Personality and Social Psychology, 78(6), 1150-1169. https://doi.org/10.1037/0022-3514.78.6.1150

Stockton, D., Kellett, S., Berrios, R., Sirois, F., Wilkinson, N., \& Miles, G. (2019). Identifying the underlying mechanisms of change during Acceptance and Commitment Therapy (ACT): A systematic review of contemporary mediation studies. Behavioural and Cognitive Psychotherapy, 47(3), 332-362. https://doi.org/10.1017/S1352465818000553

Treanor, M., Erisman, S. M., Salters-Pedneault, K., Roemer, L., \& Orsillo, S. M. (2011). An acceptance-based behavioral therapy for GAD: Effects on outcomes from three theoretical models. Depression and Anxiety, 28(2), 127-136. https://doi.org/10.1002/da.20766

Tyndall, I., Waldeck, D., Pancani, L., Whelan, R., Roche, B., \& Dawson, D. L. (2019). The Acceptance and Action Questionnaire-II (AAQ-II) as a measure of experiential avoidance: Concerns over discriminant validity. Journal of Contextual Behavioral Science, 12, 278-284. https://doi.org/10.1016/j.jcbs.2018.09.005 
Valente, M. J., \& MacKinnon, D. P. (2017). Comparing models of change to estimate the mediated effect in the pretest-posttest control group design. Structural Equation Modeling: A Multidisciplinary Journal, 24(3), 428-450. https://doi.org/10.1080/10705511.2016.1274657

Vickberg, S. M. J. (2003). The concerns about recurrence scale (CARS): A systematic measure of women's fears about the possibility of breast cancer recurrence. Annals of Behavioral Medicine, 25(1), 16-24. https://doi.org/10.1207/S15324796ABM2501_03

Weiss, D. S., \& Marmar, C. R. (1997). The Impact of Events Scale-Revised. In J. P. Wilson \& T. M. Keane (Eds.), Assessing psychological trauma and PTSD (pp. 399-411). Guilford Press.

Wilson, A. C., Mackintosh, K., Power, K., \& Chan, S. W. Y. (2019). Effectiveness of selfcompassion related therapies: A systematic review and meta-analysis. Mindfulness, 10(6), 979-995. https://doi.org/10.1007/s12671-018-1037-6

Wilson, K. G., Sandoz, E. K., Kitchens, J., \& Roberts, M. (2010). The Valued Living Questionnaire: Defining and measuring valued action within a behavioral framework. The Psychological Record, 60(2), 249. https://doi.org/10.1007/BF03395706

Wolgast, M. (2014). What does the Acceptance and Action Questionnaire (AAQ-II) really measure? Behavior Therapy, 45(6), 831-839. https://doi.org/10.1016/j.beth.2014.07.002

Zigmond, A. S., \& Snaith, R. P. (1983). The Hospital Anxiety and Depression Scale. Acta Psychiatrica Scandinavica, 67(6), 361-370. https://doi.org/10.1111/j.16000447.1983.tb09716.x 


\section{Figures}

\section{Figure 1}

Unmediated and Single Mediation Model Path Diagrams for Cancer-related Trauma Symptoms

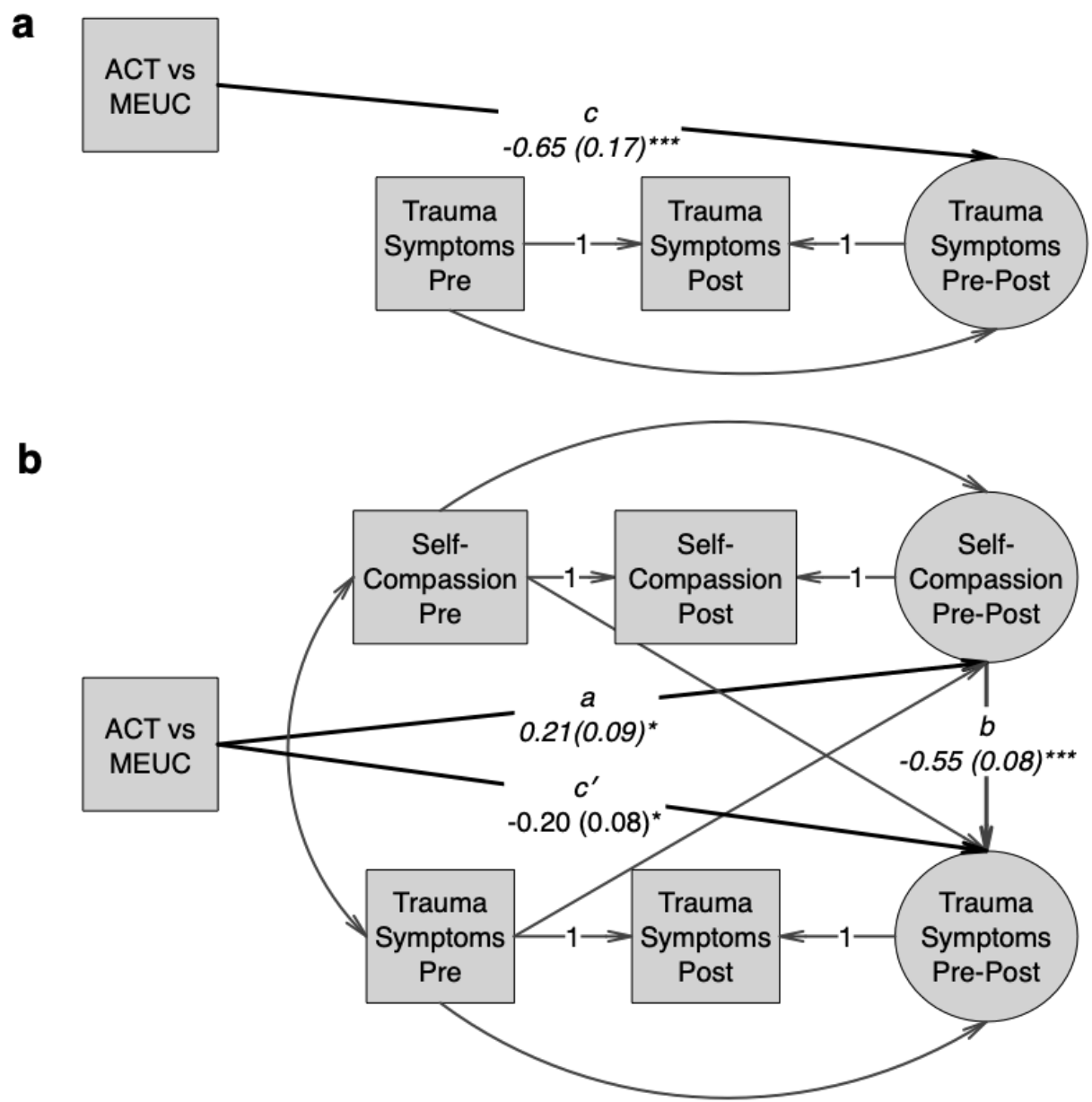

Note. Mediation models were parameterized as two timepoint latent change score models equivalent to ANCOVA models per Valente and MacKinnon (2017). Squares reflect measured variables and circles reflect latent variables. Panel A depicts the unmediated model used to compute the $c$ path and Panel B depicts a single mediator model (Table 1). Values listed under each $a, b, c$ and $c^{\prime}$ path label reflect parameter estimates with standard errors in parentheses. Unlabeled paths were freely estimated. For simplicity of presentation, variance parameters are not depicted.

$\mathrm{ACT}=$ Acceptance Commitment Therapy; $\mathrm{MEUC}=$ Minimally enhanced usual care; Pre-Post $=$ Latent difference of scores between post-intervention and pre-intervention.

$*=p<.05$

$* * *=p<.001$ 


\section{Figure 2}

Multiple Mediation Model Path Diagram for Cancer-related Trauma Symptoms

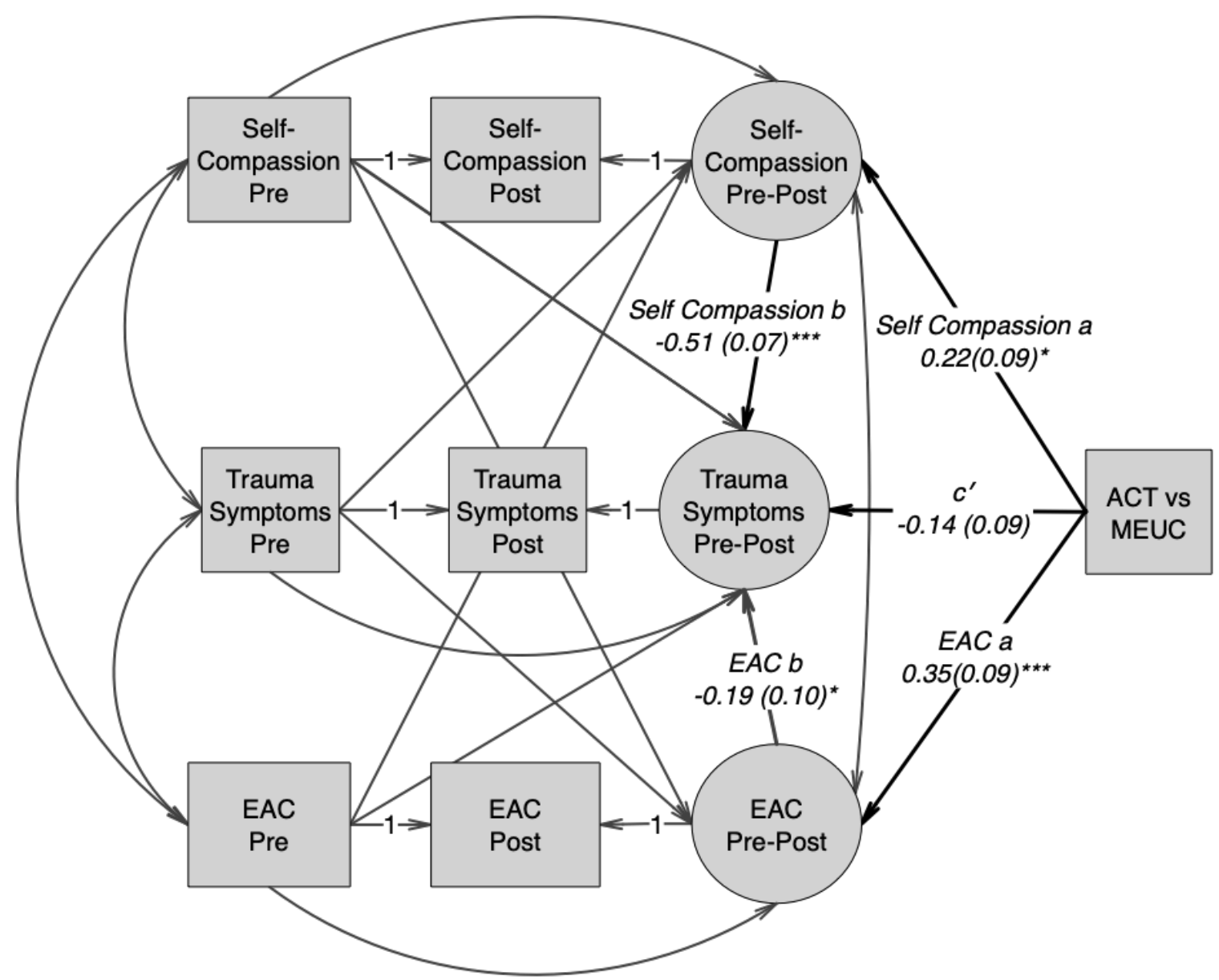

Note. The multiple mediation model was constructed based on the single mediator two-timepoint latent change score model equivalent to an ANCOVA mediation model per Valente and MacKinnon (2017). Squares reflect measured variables and circles reflect latent variables. Values listed under each $a, b$, and $c^{\prime}$ path label reflect parameter estimates with standard errors in parentheses. Unlabeled paths were freely estimated. For simplicity of presentation, variance parameters are not depicted.

ACT $=$ Acceptance Commitment Therapy; MEUC =Minimally enhanced usual care; EAC = Emotional approach coping; Pre-Post = Latent difference of scores between post-intervention and pre-intervention.

$*=p<.05$

$* * *=p<.001$ 


\section{Tables}

Table 1

Single Mediation Model Parameter Estimates

\begin{tabular}{|c|c|c|c|c|c|c|c|c|c|c|c|c|}
\hline \multirow[b]{3}{*}{ Parameter } & \multicolumn{8}{|c|}{ Core ACT Processes } & \multicolumn{4}{|c|}{ Broader ACT-Consistent Processes } \\
\hline & \multicolumn{2}{|c|}{ BEAQ } & \multicolumn{2}{|c|}{ AAQc } & \multicolumn{2}{|c|}{ Bullseye } & \multicolumn{2}{|l|}{ VLQ } & \multicolumn{2}{|c|}{ SCS } & \multicolumn{2}{|l|}{$\mathrm{EAC}$} \\
\hline & $\begin{array}{c}\text { Estimate } \\
(\mathrm{SE})\end{array}$ & $p$ & $\begin{array}{c}\text { Estimate } \\
(\mathrm{SE})\end{array}$ & $p$ & $\begin{array}{c}\text { Estimate } \\
(\mathrm{SE})\end{array}$ & $p$ & $\begin{array}{c}\text { Estimate } \\
(\mathrm{SE})\end{array}$ & $p$ & $\begin{array}{c}\text { Estimate } \\
(\mathrm{SE})\end{array}$ & $p$ & Estimate (SE) & $p$ \\
\hline \multicolumn{13}{|c|}{ Outcome $=$ Cancer-Related Trauma Symptoms (IES-R) } \\
\hline$a$ & $\begin{array}{l}-2.84 \\
(2.17)\end{array}$ & .191 & $-2.01(2.02)$ & .319 & $-2.74(1.20)$ & .023 & $2.19(2.66)$ & .411 & $\begin{array}{c}0.21 \\
(0.09)\end{array}$ & .025 & $0.35(0.09)$ & $<.001$ \\
\hline$b$ & $\begin{array}{c}0.03 \\
(0.00)\end{array}$ & $<.001$ & $0.04(0.00)$ & $<.001$ & $0.03(0.01)$ & .040 & $-0.01(0.00)$ & .141 & $\begin{array}{l}-0.55 \\
(0.08)\end{array}$ & $<.001$ & $-0.32(0.11)$ & .004 \\
\hline$c^{\prime}$ & $\begin{array}{l}-0.23 \\
(0.09)\end{array}$ & .010 & $-0.26(0.07)$ & $<.001$ & $-0.24(0.10)$ & .017 & $-0.30(0.10)$ & .002 & $\begin{array}{l}-0.20 \\
(0.08)\end{array}$ & .019 & $-0.20(0.10)$ & .038 \\
\hline$a b$ & $\begin{array}{c}-0.08 \\
(0.06)\end{array}$ & .195 & $-0.07(0.07)$ & .328 & $-0.08(0.06)$ & .159 & $-0.01(0.02)$ & .555 & $\begin{array}{c}-0.12 \\
(0.06)\end{array}$ & .037 & $-0.11(0.05)$ & .022 \\
\hline \multicolumn{13}{|c|}{ Outcome $=$ Fear of Cancer Recurrence (CARS) } \\
\hline$a$ & $\begin{array}{l}-1.87 \\
(2.23)\end{array}$ & .402 & $-1.95(1.99)$ & .325 & $-2.45(1.13)$ & .031 & $3.28(2.74)$ & .230 & $\begin{array}{c}0.19 \\
(0.10)\end{array}$ & .055 & $0.32(0.09)$ & $<.001$ \\
\hline$b$ & $\begin{array}{c}0.03 \\
(0.01)\end{array}$ & $<.001$ & $0.05(0.01)$ & $<.001$ & $0.06(0.02)$ & .002 & $0.00(0.01)$ & .540 & $\begin{array}{c}-0.75 \\
(0.14)\end{array}$ & $<.001$ & $-0.45(0.20)$ & .021 \\
\hline$c^{\prime}$ & $\begin{array}{l}-0.60 \\
(0.17)\end{array}$ & .001 & $-0.62(0.15)$ & $<.001$ & $-0.49(0.17)$ & .005 & $-0.64(0.18)$ & $<.001$ & $\begin{array}{l}-0.51 \\
(0.16)\end{array}$ & .002 & $-0.50(0.18)$ & .005 \\
\hline$a b$ & $\begin{array}{l}-0.06 \\
(0.08)\end{array}$ & .462 & $-0.09(0.10)$ & .327 & $-0.16(0.09)$ & .088 & $-0.01(0.03)$ & .686 & $\begin{array}{l}-0.14 \\
(0.08)\end{array}$ & .081 & $-0.14(0.08)$ & .065 \\
\hline
\end{tabular}

Outcome $=$ General Anxiety Symptoms (HADS-A) 


\begin{tabular}{|c|c|c|c|c|c|c|c|c|c|c|c|c|}
\hline \multirow[b]{3}{*}{ Parameter } & \multicolumn{8}{|c|}{ Core ACT Processes } & \multicolumn{4}{|c|}{ Broader ACT-Consistent Processes } \\
\hline & \multicolumn{2}{|c|}{ BEAQ } & \multicolumn{2}{|l|}{ AAQc } & \multicolumn{2}{|l|}{ Bullseye } & \multicolumn{2}{|l|}{ VLQ } & \multicolumn{2}{|c|}{ SCS } & \multicolumn{2}{|l|}{ EAC } \\
\hline & $\begin{array}{c}\text { Estimate } \\
(\mathrm{SE})\end{array}$ & $p$ & $\begin{array}{c}\text { Estimate } \\
(\mathrm{SE})\end{array}$ & $p$ & $\begin{array}{c}\text { Estimate } \\
(\mathrm{SE})\end{array}$ & $p$ & $\begin{array}{c}\text { Estimate } \\
(\mathrm{SE})\end{array}$ & $p$ & $\begin{array}{c}\text { Estimate } \\
(\mathrm{SE})\end{array}$ & $p$ & Estimate (SE) & $p$ \\
\hline$a$ & $\begin{array}{l}-2.28 \\
(2.20)\end{array}$ & .299 & $-1.87(2.03)$ & .357 & $-3.16(1.07)$ & .003 & $2.82(2.66)$ & .290 & $\begin{array}{c}0.19 \\
(0.10)\end{array}$ & .048 & $0.35(0.09)$ & $<.001$ \\
\hline$b$ & $\begin{array}{c}0.07 \\
(0.03)\end{array}$ & .005 & $0.12(0.02)$ & $<.001$ & $0.16(0.08)$ & .036 & $-0.04(0.02)$ & .085 & $\begin{array}{l}-2.20 \\
(0.34)\end{array}$ & $<.001$ & $-0.95(0.53)$ & .071 \\
\hline$c^{\prime}$ & $\begin{array}{c}-0.13 \\
(0.50)\end{array}$ & .794 & $-0.15(0.45)$ & .731 & $0.28(0.51)$ & .582 & $-0.19(0.48)$ & .698 & $\begin{array}{c}0.09 \\
(0.44)\end{array}$ & .843 & $-0.01(0.51)$ & .981 \\
\hline$a b$ & $\begin{array}{c}-0.16 \\
(0.17)\end{array}$ & .353 & $-0.23(0.25)$ & .362 & $-0.50(0.28)$ & .071 & $-0.10(0.13)$ & .446 & $\begin{array}{l}-0.41 \\
(0.24)\end{array}$ & .079 & $-0.33(0.19)$ & .086 \\
\hline
\end{tabular}

Note. Separate single mediation models, parameterized as two timepoint latent change score models equivalent to ANCOVA models per Valente and MacKinnon (2017), were estimated with each pair of outcome and mediator variables as illustrated in Figure 1b. Condition differences on within-participant differences are captured by the $a$ paths (for mediators) and $c$ paths (for outcomes).

Condition was coded as $0.5=$ Acceptance and Commitment Therapy, $-0.5=$ Minimally enhanced usual care. Estimates are derived from mediation models as depicted in Figure 1b. Standard errors (SE) were estimated by fitting the model on 10,000 nonparametric bootstrapped samples.

$\mathrm{AAQc}=$ Acceptance and Action Questionnaire-Cancer; BEAQ = Brief Experiential Avoidance Questionnaire; CARS $=$ Concerns About Recurrence Scale; EAC = Emotional Approach Coping scale; HADS-A = Hospital Anxiety and Depression Scale-Anxiety subscale; IES-R = Impact of Events Scale-Revised total score; SCS = Self-Compassion Scale-Short Form; VLQ = Valued Living Questionnaire. 


\section{Table 2}

Cancer-Related Trauma Symptom (IES-R) Multiple Mediation Model Estimates

\begin{tabular}{cccc}
\hline Parameter & Estimate & SE & $p$ \\
\hline Self-Compassion $a$ & 0.22 & 0.09 & .018 \\
$c^{\prime}$ & 0.35 & 0.09 & $<.001$ \\
EAC $a$ & -0.14 & 0.09 & .113 \\
EAC $b$ & -0.51 & 0.07 & $<.001$ \\
Self-Compassion $a b$ & -0.11 & 0.05 & .030 \\
EAC $a b$ & -0.07 & 0.04 & .089 \\
Total $a b$ & -0.18 & 0.07 & .008 \\
\hline
\end{tabular}

Note. Two target processes, self-compassion and emotional approach coping, were entered as simultaneous mediators of change in cancer-related trauma symptoms (see Figure 2) because both of those target processes were significant mediators of the cancer-related trauma symptoms outcome in single mediation models (see Table 1). The multiple mediation model was constructed based on the single mediator two-timepoint latent change score model equivalent to an ANCOVA mediation model per Valente and MacKinnon (2017). Standard errors (SE) were estimated by fitting the model on 10,000 nonparametric bootstrapped samples. $\mathrm{EAC}=$ Emotional Approach Coping scale; IES-R = Impact of Events Scale-Revised total score; $\mathrm{SCS}=$ Self-Compassion Scale-Short Form. 